Acta Botanica Mexicana (2003), 64: 1-18

\title{
ESPECIES DE STENOCEREUS CON ARÉOLAS MORENAS: CLAVE Y DESCRIPCIONES
}

\author{
Hilda J. Arreola-Nava y Teresa Terrazas \\ Programa en Botánica, Colegio de Postgraduados \\ 56230 Montecillo, Estado de México, México
}

\begin{abstract}
RESUMEN
Se presenta una clave y las descripciones de las especies de Stenocereus que conforman el grupo con aréolas morenas, basadas en la revisión de ejemplares de herbario, observaciones en el campo y colecta intensiva. De acuerdo con este trabajo, son siete las especies de Stenocereus con aréolas morenas y se distinguen de los otros representantes conocidos del género por sus tricomas glandulares que le confieren el color obscuro a todas sus aréolas y porque su distribución se centra en el occidente de México.
\end{abstract}

Palabras clave: Cactaceae, México, Stenocereus.

\begin{abstract}
A key and descriptions of Stenocereus species with brown areoles are presented based on the revision of herbarium specimens, field observations, and intensive field collections. The species of Stenocereus with brown areoles are seven and can be distinguished from other species of the genus up to day known in their glandular trichomes conferring a dark color to all their areoles and in their distribution centered in western Mexico.
\end{abstract}

Key words: Cactaceae, Mexico, Stenocereus.

\section{INTRODUCCIÓN}

Stenocereus es el género de más amplia distribución de la tribu Pachycereae de la familia Cactaceae (Barthlott y Hunt, 1993), incluye 23 especies de las cuales la mayoría son endémicas de México (Hunt, 1999). A este taxon pertenecen las plantas conocidas como pitayas, cuyos frutos se aprovechan para el consumo humano. Todas habitan en el bosque tropical caducifolio y en el matorral xerófilo del país. Gibson y Horak (1978) reconocieron varias especies a las que llamaron "Stenocereus con aréolas morenas", porque tienen tricomas glandulares en las aréolas del tallo y flores, en contraste con los otros componentes de Stenocereus que carecen de los mismos. La presencia de tricomas glandulares es una peculiaridad no sólo dentro del género sino en la subfamilia Cactoideae. 
En diferentes trabajos varía el número de especies incluidas en el grupo; Gibson et al. (1986) señalan que lo conforman nueve especies: S. beneckei (Ehrenb.) Buxb., S. chrysocarpus Sánchez-Mej., S. fricii Sánchez-Mej., S. littoralis (K. Brandegee) L. W. Lenz, S. montanus (Britton et Rose) Buxb., S. queretaroensis (F. A. C. Weber) Buxb., S. quevedonis (J. G. Ortega) Bravo, S. pruinosus (Otto ex Pfeiff.) Buxb. y S. thurberi (Engelm.) Buxb.; más tarde Gibson (1990) añade a S. chacalapensis (Bravo et T. MacDoug.) Buxb. y a S. martinezii (J. G. Ortega) Bravo y excluye a S. fricii y S. pruinosus. Recientemente, Arreola-Nava (2000) propone redefinir el grupo de aréolas morenas con base en los resultados de un análisis filogenético e incluye únicamente siete entidades: Stenocereus beneckei, S. chrysocarpus, S. martinezii, S. montanus, S. queretaroensis, S. quevedonis y $S$. thurberi. Estas especies conforman un clado monofilético, a exclusión de $S$. chacalapensis, $S$. fricii, $S$. littoralis y $S$. pruinosus. Algunas de las especies de Stenocereus con aréolas morenas no habían sido descritas en todas sus estructuras, sobre todo las florales, y no existía material de referencia adecuado que permitiera compararlas, por lo que a menudo se habían confundido entre ellas, en especial $S$. chrysocarpus, $S$. montanus y $S$. queretaroensis. En tal circunstancia se consideró pertinente presentar la clave y la descripción de los caracteres morfológicos de cada una.

Se revisaron los ejemplares depositados en ocho herbarios: ARIZ, CHAPA, ENCB, IBUG, IEB, MEXU, RSA y SD, incluyendo los especímenes tipo depositados en MEXU. Además se realizaron colectas y observaciones en varias poblaciones a lo largo del área de distribución de las especies, visitando preferentemente las localidades durante las épocas de floración y fructificación.

\section{CLAVE PARA LAS ESPECIES DE STENOCEREUS CON ARÉOLAS MORENAS}

1. Ramas sin constricción horizontal debajo de la aréola; espinas del tallo no claramente definidas en radiales y centrales; fruto con espinas de color amarillo a amarillo intenso.

2. Podarios del pericarpelo y tubo receptacular poco prominentes, con espinas fuertes y bien desarrolladas ................................................ S. montanus

2. Podarios del pericarpelo y tubo receptacular muy tuberculados, con espinas suaves y setosas.

3. Árboles con tronco muy largo, de 1.5 a $3 \mathrm{~m}$; flores de más de $8.8 \mathrm{~cm}$ de largo; pericarpelo y tubo receptacular con tricomas numerosos muy densos y abundantes

S. chrysocarpus

3. Árboles con tronco de 0.5 a $1.2 \mathrm{~m}$; flores de 7.8 a $8.4 \mathrm{~cm}$ de largo; pericarpelo y tubo receptacular con tricomas poco densos ......... S. queretaroensis

1. Ramas con una constricción horizontal debajo de la aréola; espinas del tallo claramente definidas en radiales y centrales; fruto con espinas blancas, blancorojizas o negruzcas.

4. Arbustos menores de $2 \mathrm{~m}$ de alto; ramas erectas o decumbentes de color verde-azuloso a grises S. beneckei 
4. Árboles de 3 m o más de alto; ramas erectas de color verde obscuro a verde claro.

5. Espinas blancas; cavidades de mucílago no evidentes en corte transversal del tallo; fruto con espinas blancas ................................ S. quevedonis

5. Espinas negras; cavidades de mucílago evidentes en corte transversal del tallo; fruto con espinas blanco-rojizas a negruzcas

6. Costillas de 12 a 21; espinas radiales rectas y aciculares

S. thurberi

6. Costillas de 10 a 12; espinas radiales ligeramente recurvadas y no aciculares S. martinezii

\section{DESCRIPCIONES DE LAS ESPECIES}

Stenocereus beneckei (Ehrenb.) Buxb., Bot. Stud. 12: 92. 1961.

Cereus beneckei Ehrenb., Bot. Zeit. 2: 835. 1844.

Cereus beneckei farinosus Salm-Dyck, Cact. Hort. Dyck. 1849: 48.1850.

Pipthanthocereus beneckei (Ehrenb.) Riccob., Boll. R. Ort. Bot. Palermo 8: 226. 1909.

Lemaireocereus beneckei (Ehrenb.) Britton et Rose, Cactaceae 4: 273. 1923.

Hertrichocereus beneckei (Ehrenb.) Backeb., Cact. Succ. J. (USA) 22: 153. 1950.

Tipo: México, sobre cama de lava; sin localidad, s/n, s/f (tipo no localizado). Neotipo (aquí designado): México, Guerrero, mpio. Taxco, carr. Iguala - Taxco a $10 \mathrm{~km}$ de Iguala y $400 \mathrm{~m}$ de Taxco El Viejo, T. Terrazas y C. Catalán 466 (IBUG).

Arbustos poco ramificados, 1-2 m de alto; tronco no definido; ramas erectas o decumbentes, 5-7 cm de diámetro, verde-azulosas a grises, las ramas jóvenes farinosas; cavidades de mucílago no evidentes en corte transversal del tallo; costillas 6-9, fuertemente tuberculadas, redondeadas a obtusas en sección transversal, asimétricas en sección longitudinal, $1-1.5 \mathrm{~cm}$ de alto, con una constricción horizontal entre las aréolas de la misma costilla; aréolas situadas en la parte apical de los tubérculos, circulares, pequeñas, 4-5 mm de diámetro, las jóvenes con tricomas glandulares pardos que con el tiempo se vuelven negros; espinas no se observan a simple vista, pero cuando se ven son 1-3, una de ellas, la superior, acicular, recta, rígida, de cerca de $5 \mathrm{~cm}$ de largo, al principio negra, después grisácea, las inferiores 0.3-1.7 cm de largo, aciculares, negras, con el tiempo se vuelven grisáceas o se caen; en las aréolas maduras las espinas son numerosas, están claramente definidas en radiales y centrales; flores sólo en la región apical de los tallos, una por aréola, nocturnas, angostamente infundibuliformes, 5.5-6.8 cm de largo y 2.9$4.9 \mathrm{~cm}$ de ancho en antesis; pericarpelo con pocos tubérculos pero prominentes, pequeños, globosos, alrededor de $6 \mathrm{~mm}$ de diámetro, de color verde-azuloso a gris, aréolas en el ápice de los tubérculos, con primordios foliares y escamosos, 
apiculados, axilas con escasos tricomas glandulares marrón-rojizos, con espinas suaves, setosas y rojizas; tubo receptacular alrededor de $4 \mathrm{~cm}$ de largo, angosto, con podarios escasos y altos, espinas suaves y setosas; segmentos exteriores del perianto espatulados, de color verde claro con tintes rojizos, margen entero a ciliado en el ápice; segmentos interiores espatulados, blanco-verdosos, margen ciliado de color blanco-amarillento, con algunos tonos verdosos; estambres inclusos, numerosos, insertos en series verticiladas; filamentos blancos; anteras basifijas, de color crema; estilo blanco-amarillento; lóbulos del estigma 6-8, 3-5 mm de largo, blanco-amarillentos; cámara nectarial semicerrada por la curvatura de los estambres del verticilo inferior, muy larga, $1.6-2.4 \mathrm{~cm}$ de largo y $0.28-0.38 \mathrm{~cm}$ de ancho, de paredes estriadas; ovario $0.5-0.6 \mathrm{~cm}$ de largo y $0.4-0.6 \mathrm{~cm}$ de ancho; fruto elíptico, con tubérculos muy prominentes, menor de $3 \mathrm{~cm}$ de largo, al principio verdepardusco, más tarde rojo, aréolas de los tubérculos con una escama pequeña y 8-9 espinas rígidas blanco-rojizas a negruzcas, hasta $2 \mathrm{~cm}$ de largo; semillas 3.1$3.3 \mathrm{~mm}$ de largo y 2.3-2.5 $\mathrm{mm}$ de ancho, de forma lenticular u ovoide, negras, brillantes, células de la testa isodiamétricas, sus paredes periclinales planas, con intersticios entre las paredes anticlinales, lo que le confiere un aspecto ligeramente punteado.

Fenología. Florece de octubre a diciembre, fructifica de enero a marzo.

Hábitat. Crece en bosque tropical caducifolio, en altitudes de los 1200 a 1400 m. Convive con Neobuxbaumia mezcalaensis (Bravo) Backeb., Pachycereus weberi (Coult.) Backeb. y Stenocereus pruinosus.

Distribución. Stenocereus beneckei centra su distribución en Morelos, Guerrero, Puebla y el Estado de México (Fig. 1).

Ejemplares examinados. Morelos: mpio. Ayala, Villa de Ayala, Cuautla, $D$. B. Gold s/n (MEXU); mpio. Tepalzingo, El Limón, B. Guerrero C. 1343 (MEXU). Estado de México: mpio. Ixtapan de la Sal, Barranca de Calderón, cerca de Ixtapan de la Sal, H. Bravo s/n (MEXU). Puebla: mpio. Acatlán de Osorio, $1 \mathrm{~km}$ al oeste de Petlalcingo, U. Guzmán, S. Gama y S. Arias 881 (MEXU). Guerrero: mpio. Chilpancingo, entre Río Mezcala y Chilpancingo, L. Scheinvar y H. Bravo 735 (MEXU); mpio. Chilpancingo, Río Mezcala, L. Scheinvar y cols. 5352 (MEXU); mpio. Taxco, carretera Iguala - Taxco a $10 \mathrm{~km}$ de Iguala y $400 \mathrm{~m}$ de Taxco El Viejo, $T$. Terrazas y C. Catalán 466 (IBUG), 468 (CHAPA); mpio. Taxco, Cerro de Acuitlapan, cerca de Taxco, F. Castañón s/n (MEXU); mpio. Taxco, km 106 turnoff to Temaxcalapa on Highway 95 (Iguala to Taxco), A. C. Gibson y B. Prigge 3716 (RSA); mpio. Taxco, cerca de las Grutas de Cacahuamilpa, H. Bravo s/n (MEXU).

Stenocereus chrysocarpus Sánchez-Mej., Cact. Suc. Mex. 17: 95. 1972.

Tipo: México, Michoacán, mpio. Arteaga, Palo Pintado. H. Sánchez-Mejorada 71-0503 (MEXU!). 
Árboles candelabriformes, 5-9 m de alto; tronco bien definido 1.5-3 m de alto y $25-40 \mathrm{~cm}$ de diámetro; ramas abundantes, paralelas, no muy cercanas, $2-5 \mathrm{~m}$ de largo y 10-14 cm de diámetro, de color verde claro, a veces con ramificaciones secundarias y terciarias; costillas comúnmente 7 , a veces 8 , rectas, ligeramente serruladas, agudas en sección transversal, $3.5-4 \mathrm{~cm}$ de alto, $1-1.5 \mathrm{~cm}$ de ancho en la base, distantes 3.5-6 cm entre sus bordes; aréolas situadas en los ápices comprimidos de los podarios, transversalmente elípticas, 4-5 mm de largo y 8-10 mm de ancho, con tricomas glandulares de color rojizo que después se tornan pardos a negruzcos, a ambos lados de la aréola se observa una depresión entre los podarios de ca. de $3 \mathrm{~mm}$ de longitud; espinas radiales en el borde de la aréola excepto en la parte superior, semirradiantes, descendentes, casi horizontales por lo comprimido de la aréola, aciculares, rígidas, rectas, blancas con punta amarillenta cuando jóvenes, grisáceas después, la inferior más larga, 1.1-1.5 cm de longitud; espinas centrales $0-2$, aciculares, rígidas, rectas, a veces $2 \mathrm{~mm}$ más cortas o más largas que las radiales, reflejas, surgen en la parte inferior de la aréola; flores en el cuarto superior de las ramas, nocturnas, que permanecen abiertas hasta la mañana siguiente, infundibuliformes, $8.8-10.6 \mathrm{~cm}$ de largo, ancho del perianto 5.3$7.7 \mathrm{~cm}$; pericarpelo ovoide, $2-3 \mathrm{~cm}$ de largo y alrededor de $2.5 \mathrm{~cm}$ de diámetro, provisto de tubérculos prominentes, imbricados, verdes con primordios foliares triangulares, pequeños, de color moreno y con aréolas en las axilas, alrededor de $2 \mathrm{~mm}$ de diámetro, con tricomas amarillos numerosos muy densos y abundantes, con varias espinas amarillentas, suaves y setosas de hasta de $8 \mathrm{~mm}$ de longitud; tubo receptacular 2.8-3.6 cm de largo, con escamas, podarios prominentes, alargados y decurrentes que tienen una escama foliar en el ápice y algunas espinas amarillentas, suaves y setosas; segmentos exteriores del perianto apiculados, verde-rojizos, alrededor de $2.5 \mathrm{~cm}$ de largo y de $1.5 \mathrm{~cm}$ de ancho; segmentos interiores oblongos, con el ápice obtuso, de margen ligeramente ciliado, blancos o a veces amarillentos, de aproximadamente $2 \mathrm{~cm}$ de largo y $0.8-1 \mathrm{~cm}$ de ancho; estambres inclusos, numerosos, insertos en series verticiladas, alrededor de $1.6 \mathrm{~cm}$ de longitud; filamentos blanco-rosados; anteras basifijas, amarillo-rosadas; estilo blanco, 5-6 cm de largo; lóbulos del estigma 8-10, blanco-rosados, 0.9-1.1 cm de largo; cámara nectarial semicerrada por la curvatura de los estambres del verticilo inferior, $1.4-1.8 \mathrm{~cm}$ de largo y $0.8-1 \mathrm{~cm}$ de ancho, estriada; ovario $1.1-1.7 \mathrm{~cm}$ de largo y 0.7-0.9 cm de ancho; fruto elíptico, alrededor de $6 \mathrm{~cm}$ de largo por $4 \mathrm{~cm}$ de diámetro, rojo-purpúreo, provisto de pequeñas escamas cuyas axilas Ilevan aréolas circulares, alrededor de $6 \mathrm{~mm}$ de diámetro, con tricomas abundantes, de color amarillo-paja y numerosas espinas aciculares, delgadas, setosas, $12-20 \mathrm{~mm}$ de longitud, de color amarillo-paja, divergentes y entrelazadas cubriendo por completo el fruto, aréolas del fruto caducas desprendiéndose al madurar, pulpa jugosa, dulce, de color rojo-escarlata a solferino; semillas alrededor de $2.5 \mathrm{~mm}$ de largo y $1.8 \mathrm{~mm}$ de ancho, ovoides, negras, opacas, células de la testa isodiamétricas con paredes periclinales evidentemente convexas, sin intersticios entre las paredes anticlinales, lo que le confiere a la semilla un aspecto finamente verrucoso. 
Fenología. Florece en primavera durante los meses de marzo y abril, fructifica en agosto y septiembre.

Hábitat. Bosque tropical caducifolio en altitudes entre los 500 y 700 m.

Distribución. Stenocereus chrysocarpus habita en la cuenca del río Balsas en Michoacán, así como en Guerrero (Fig. 1). Otras cactáceas columnares que se encuentran conviviendo con ella son S. fricii, S. quevedonis, S. standleyi (J. G. Ortega) Buxb., Pachycereus marginatus (DC.) Britton et Rose, P. militaris (Audot) Hunt y P. tepamo S. Gama et S. Arias.

Ejemplares examinados. Michoacán: mpio. Apatzingán, carretera ApatzingánBuenavista a $3 \mathrm{~km}$ al sur del poblado El Cinco, H. J. Arreola-Nava, T. Terrazas y S. Arias 1578 (CHAPA), 1579 (CHAPA); mpio. Arteaga, al pie de lomas situadas unos $10 \mathrm{~km}$ al NW de El Infiernillo, H. Sánchez-Mejorada 2713 (MEXU), mpio. Arteaga, Palo Pintado, S. Arias y cols. 707 (MEXU); mpio. Arteaga, Palo Pintado on SW edge of town at km 224-225. A. C. Gibson y B. Prigge 3716 (RSA); mpio. Arteaga, carretera a Playa Azul, en el poblado Palo Pintado, H. J. Arreola-Nava, T. Terrazas, S. Arias y R. Herrera 1522 (CHAPA), 1524 (CHAPA), 1525 (CHAPA), 1529 (CHAPA); mpio. Arteaga, $2.3 \mathrm{~km}$ del poblado Palo Pintado sobre la carretera Pátzcuaro - Playa Azul, H. J. Arreola-Nava, T. Terrazas y S. Arias 1575 (CHAPA); mpio. Arteaga, Rancho La Parota, Las Juntas, cerca del Infiernillo, L. Scheinvar 2161 (CHAPA); mpio. Tepalcatepec, $10 \mathrm{~km}$ al SW de Tepalcatepec, S. Arias y cols. 692 (MEXU), 694 (MEXU). Guerrero: mpio. Coyuca de Catalán, El Cundancito, L. Scheinvar y cols. 5382 (MEXU), 5383 (MEXU); mpio. Coyuca de Catalán, El Cundancito, S. Arias y cols. 715 (MEXU).

Stenocereus martinezii (J. G. Ortega) Bravo, Cact. Suc. Mex. 17: 119. 1972. Lemaireocereus martinezii J. G. Ortega, Apuntes para la Flora Indígena de Sinaloa. 1929.

Tipo: México, sin localidad (no designado). Lectotipo (aquí designado), el ejemplar corresponde a la colección del autor y a la zona de la que se describió la especie (González-Ortega, 1929): México, Sinaloa, mpio. Mazatlán. El Quelite. s/f, J. González-Ortega 5542 (MEXU).

Árboles candelabriformes, 3-5 m de alto; tronco definido, 1.0-1.5 m de largo y $25-30 \mathrm{~cm}$ de diámetro; ramas erectas, escasas, a veces volviéndose a ramificar, casi todas de la misma longitud, de hasta $2.5 \mathrm{~m}$ de largo y $20 \mathrm{~cm}$ de diámetro, verdes oscuras; cavidades de mucílago evidentes en corte transversal del tallo; costillas 10-12, en sección longitudinal simétricas, en sección transversal predominantemente redondeadas, $1.5-2.5 \mathrm{~cm}$ de alto y $2-3.5 \mathrm{~cm}$ de ancho en la base, con una constricción horizontal entre las aréolas de la misma costilla; aréolas 
distantes entre sí 1.5-3 cm, grandes, 7-10 mm de diámetro, circulares, con tricomas glandulares que le dan un aspecto rojo-pardusco en las aréolas jóvenes y cuyo exudado forma con el tiempo una costra negra; espinas radiales 7-11, 2-10 mm de largo, negras, blanco-grisáceas con la edad, las superiores y laterales muy cortas, las inferiores más largas que las demás, no aciculares, subuladas, ligeramente recurvadas; espinas centrales 3 o a veces sólo una, subuladas, la inferior más larga que las demás, ligeramente curva, refleja, de hasta $3 \mathrm{~cm}$ de largo, negras; flores en la región subapical o lateral, nocturnas, abiertas hasta la mañana siguiente, infundibuliformes, 5-7 cm de largo y 3.1-6.6 cm de ancho en la antesis; pericarpelo globoso, de color verdoso con tintes rojizos, $1-1.5 \mathrm{~cm}$ de diámetro, cubierto por podarios prominentes imbricados con primordios foliares cortos, alrededor de $2 \mathrm{~mm}$ de largo y $2 \mathrm{~mm}$ de ancho en la base, triangulares, verdes con la punta purpúrea, con tricomas blanco-amarillentos en las axilas y algunas espinas setosas, rojizas, de hasta de $2.6 \mathrm{~mm}$ de largo; tubo receptacular $1-1.5 \mathrm{~cm}$ de largo, podarios con escamas decurrentes, oblongas, con el ápice agudo a apiculado, de longitud variable, alrededor de $3 \mathrm{~mm}$ de ancho, con algunas espinas setosas, rojizas; segmentos exteriores del perianto angostamente obovados a espatulados, con el ápice redondeado a agudo o mucronado, margen entero o ligeramente ciliado, en el haz de color blanco-amarillento, en el envés verdes claros con tintes purpúreos, $1-2 \mathrm{~cm}$ de largo y $0.6-0.8 \mathrm{~cm}$ de ancho; segmentos interiores oblongos a obtusos, alrededor de $1.8 \mathrm{~cm}$ de largo y $0.8 \mathrm{~cm}$ de ancho, de borde entero, blancoamarillentos; estambres inclusos, numerosos, insertos en el tubo receptacular, en series verticiladas; filamentos amarillentos; anteras basifijas, blanco-amarillentas; estilo $4-4.5 \mathrm{~cm}$ de largo y alrededor de $2 \mathrm{~mm}$ de ancho; lóbulos del estigma 8-11, $0.55-0.65 \mathrm{~cm}$ de largo, blanco-amarillentos; cámara nectarial semicerrada por la curvatura de los estambres del verticilo inferior, 0.9-1.1 cm de largo y 4.9-5.9 mm de ancho, estriada; ovario $0.5-0.7 \mathrm{~cm}$ de largo y $0.6-0.8 \mathrm{~cm}$ de ancho; fruto globoso a ovoide, dehiscente en la madurez, verde a verde-rojizo, 3.0-4.5 cm de diámetro, cubierto por aréolas con tricomas blanco-amarillentos y numerosas espinas rígidas, de hasta $2.5 \mathrm{~cm}$ de largo, blanco-rosadas a blanco-rojizas, caducas en la madurez, pulpa roja, dulce; semillas 1.8-2 mm de largo y 1.2-1.4 mm de ancho, ovoides, negras, opacas, células de la testa isodiamétricas, sus paredes periclinales convexas, sin intersticios, lo que le confiere a la semilla un aspecto finamente verrucoso.

Fenología. Florece durante abril y mayo, fructifica en mayo y junio.

Hábitat. Bosque tropical caducifolio, en altitudes cercanas a los $100 \mathrm{~m}$.

Distribución. Stenocereus martinezii está restringida al centro y sur del estado de Sinaloa (Fig.1). Convive con Pachycereus pecten-aboriginum (Engelm.) Britton et Rose y Stenocereus alamosensis (Coult.) A. C. Gibson et K. E. Horak. 
Ejemplares examinados. Sinaloa: mpio. Mazatlán, Sindicatura de El Quelite, La Ramada, J. González-Ortega 5542 (MEXU); mpio. Culiacán, carretera federal Culiacán - Mazatlán, km 166 frente a la cementera, H. J. Arreola-Nava, T. Terrazas y S. Arias 1560 (CHAPA); mpio. Culiacán, carretera federal Culiacán - Mazatlán km 166, desviación a Sta. Cruz de Ayala antes de pasar el Río San Lorenzo y el poblado de Tabala, H. J. Arreola-Nava, T. Terrazas y S. Arias 1561 (CHAPA), 1562 (CHAPA), 1563 (CHAPA); mpio. Elota, carretera federal Culiacán - Mazatlán, km 90, H. J. Arreola-Nava, T. Terrazas y S. Arias 1564 (CHAPA), 1566 (CHAPA); mpio. Elota, carretera federal Culiacán - Mazatlán, km 100, E. Villegas F., H. J. ArreolaNava y S. Arias 517 (IBUG).

Stenocereus montanus (Britton et Rose) Buxb., Bot. Stud. 12: 92.1961. Lemaireocereus montanus Britton et Rose, The Cactaceae 2: 97. 1920. Ritterocereus montanus (Britton et Rose) Backeb., Cact. Succ. J. (USA) 23: 121. 1951.

Tipo: México, Sonora, Álamos. J. N. Rose, P. C. Standley y P. G. Russell 13039 (US).

Árboles candelabriformes, 6-9 $\mathrm{m}$ de alto; tronco definido, 0.6-1 $\mathrm{m}$ de alto y 30-40 cm de diámetro; ramas más o menos numerosas que se vuelven a ramificar en secundarias y terciarias, encorvadas en la base, casi todas del mismo tamaño, 1-5 m de largo y 13-20 cm de diámetro, verdes oscuras; costillas 7-9, agudas en sección transversal, rectas en sección longitudinal, de aproximadamente $2.7 \mathrm{~cm}$ de altura y $4 \mathrm{~cm}$ de ancho en la base, sin constricción horizontal entre aréolas de la misma costilla; aréolas distantes entre sí $1-1.5 \mathrm{~cm}, 6-11 \mathrm{~mm}$ de largo y 6-10 mm de ancho, circulares a elípticas en sección transversal, con tricomas glandulares de color marrón claro, con el tiempo forman una costra de color marrón oscuro a negro; espinas no claramente diferenciadas en radiales y centrales, 9-10, 0.5-1.5 cm de largo, una de ellas, la inferior, más larga que las demás, de hasta $3 \mathrm{~cm}$ de largo, aciculares a subuladas, divergentes, fuertes, blancas, con el tiempo grises, la inferior refleja; flores subapicales o laterales, nocturnas, infundibuliformes, $6.6-8.2 \mathrm{~cm}$ de largo y $3.5-5 \mathrm{~cm}$ de ancho; pericarpelo ovoide, $1-1.8 \mathrm{~cm}$ de largo y $1-1.5 \mathrm{~cm}$ de ancho, verde con tintes purpúreos, con podarios no prominentes, con primordios foliares triangulares, cortos, 2-3 mm de largo y ancho en la base, purpúreo-verdosos con los bordes purpúreos, provistos en las axilas de tricomas blanco-amarillentos, espinas fuertes, bien desarrolladas; tubo receptacular $3-3.5 \mathrm{~cm}$ de largo, podarios con escamas soldadas a él, oblongas con el ápice agudo a mucronado, de longitud variable y 3-8 $\mathrm{mm}$ de ancho, con espinas fuertes; segmentos exteriores del perianto angostamente obovados a espatulados, con el ápice agudo a redondeado, mucronado, alrededor de $2.5 \mathrm{~cm}$ de largo y $1 \mathrm{~cm}$ de ancho, en el haz de color verdeamarillento, en el envés verde con tintes purpúreos; segmentos interiores oblongos a espatulados con el ápice apiculado, borde entero, blancos a blanco-rosados, alrededor de $2.5 \mathrm{~cm}$ de largo y $1 \mathrm{~cm}$ de ancho; estambres numerosos, insertos en 
el tubo receptacular en series verticiladas; filamentos amarillentos; anteras basifijas, blanco-amarillentas; estilo blanco-amarillento, alrededor de $2 \mathrm{~mm}$ de ancho; lóbulos del estigma 8-10, 6.5-8.5 mm de largo, blanco-amarillentos; cámara nectarial semicerrada por la curvatura de los estambres del verticilo inferior, $1.2-1.6 \mathrm{~cm}$ de largo y $4.6-6.8 \mathrm{~mm}$ de ancho, estriada; ovario $0.9-1.3 \mathrm{~cm}$ de largo y $0.6-0.8 \mathrm{~cm}$ de ancho; fruto 5-6 cm de diámetro, globoso a ovoide, verde a verde-rojizo o de color púrpura, cubierto por aréolas con numerosas espinas setosas, alrededor de $1.5 \mathrm{~cm}$ de largo, de color amarillo pálido, caducas en la madurez, pulpa roja, de sabor agradable; semillas 2.3-2.5 mm de largo y 1.62-1.78 $\mathrm{mm}$ de ancho, ovoides, negras, opacas, células de la testa isodiamétricas, con paredes periclinales convexas, sin intersticios lo que le confiere un aspecto finamente verrucoso.

Fenología. Florece en abril y mayo, fructifica en mayo y junio.

Hábitat. Bosque tropical caducifolio en altitudes entre los 200 y 400 m.

Distribución. Stenocereus montanus se encuentra en el sur de Sonora y norte de Sinaloa (Fig. 1). Cohabita con otras cactáceas columnares como Pachycereus pecten-aboriginum y $S$. thurberi.

Ejemplares examinados. Sonora: mpio. Álamos, El Guayabo crossing of Río Cuchujaqui, $2.6 \mathrm{~km}$ northeast of Sabinito Sur, $14 \mathrm{~km}$ southeast Álamos, T. R. Van Devender, S. L. Friedman, C. J. Davis y R. Figueroa 94-152 (ARIZ); mpio. Álamos, Arroyo Las Bebelamas, $2 \mathrm{~km}$ northeast of Rincón Viejo, $5 \mathrm{~km}$ north of Álamos, $T$. R. Van Devender, D. A. Yetman, R. A. López-Estudillo y F. Valenzuela 94231(ARIZ); mpio. Álamos, El Guayabo crossing of Río Cuchujaqui, $2.6 \mathrm{~km}$ northeast of Sabinito Sur, $14 \mathrm{~km}$ southeast Álamos, T. R. Van Devender, S. A. Meyer, J. F. Wiens, C. D. Bertelsin y K. J. Johnson 93-183 (ARIZ); mpio. Álamos, Los Tanques, cerro al sur del poblado sobre camino de terracería a $800 \mathrm{~m}$ del pueblo, $H$. J. ArreolaNava, T. Terrazas y S. Arias 1547 (CHAPA); mpio. Álamos, camino de terracería entre Tezcalama - Cochibampo, $37.4 \mathrm{~km}$ de Alamos, H. J. Arreola-Nava, T. Terrazas y S. Arias 1549 (CHAPA); mpio. Álamos, camino a Cochibampo, laderas antes de entrar al poblado km 40.6, H. J. Arreola-Nava, T. Terrazas y S. Arias 1550 (CHAPA); mpio. Álamos, La Aduana (west of Álamos) foothills, R. S. Felger y A. Russell 20333 (ARIZ). Sinaloa: mpio. Ahome, carretera Los Mochis - Topolobampo a $4 \mathrm{~km}$ de Los Mochis, H. J. Arreola-Nava, T. Terrazas y S. Arias 1559 (CHAPA); mpio. Choix, carretera El Fuerte - Choix a $28 \mathrm{~km}$ de El Fuerte, en la desviación a Antonio Canales, H. J. Arreola-Nava, T. Terrazas y S. Arias 1556 (CHAPA), 1557 (CHAPA).

Stenocereus queretaroensis (F. A. C. Weber) Buxb., Bot. Stud. 12: 92.1961. Cereus queretaroensis F. A. C. Weber, in Mathsson, Monts. Kakt. 1: 27. 1891. Pachycereus queretaroensis (F. A. C. Weber) Saff., Ann. Rep. Smiths. Inst. 1908. PI. 6. f. 2. 1909.

Ritterocereus queretaroensis (F. A. C. Weber) Backeb., Cact. Succ. J. (USA) 23: 121. 1951. 
Tipo: México, Querétaro, s/n, s/f (no localizado). Neotipo (aquí designado): México, Querétaro, mpio. Querétaro, km 8 camino rumbo a Tlacote, desde el entronque con la carretera Querétaro - San Luis Potosí, H. J. Arreola-Nava, A. Orduño y M. A. Martínez-Colín 1519 (IBUG).

Árboles candelabriformes, 3-7 $\mathrm{m}$ de alto; tronco definido, 0.5-1.2 m de alto y $20-25 \mathrm{~cm}$ de diámetro o más; ramas principales dividiéndose en ramas secundarias y terciarias y formando una copa muy amplia, de tamaños variables de hasta $4 \mathrm{~m}$ y aproximadamente $15 \mathrm{~cm}$ de diámetro, verdes, a veces con tintes rojizos; costillas 7-9, agudas en sección transversal, rectas en sección longitudinal, alrededor de $3 \mathrm{~cm}$ de altura y de $5 \mathrm{~cm}$ de ancho en la base, sin constricción horizontal entre las aréolas; aréolas distantes entre sí 1-3 cm, elípticas, 4-10 mm de largo y 6-9 mm de ancho, con tricomas glandulares de color marrón claro, que con el tiempo le confieren a la aréola un color marrón oscuro a negro y forman una costra sobre la misma; espinas radiales 5-6, emergiendo en la mitad inferior de la aréola, subuladas, desiguales, $0.5-3 \mathrm{~cm}$ de largo, blancas con la base rojiza cuando jóvenes, grisáceas con la edad, las inferiores reflejas; espinas centrales generalmente ausentes, a veces 1-2, aciculares, de hasta $3 \mathrm{~cm}$ de largo, blancas con la base rojiza cuando jóvenes, después blancas a grises; flores laterales 0 subapicales, nocturnas que permanecen abiertas durante la mañana siguiente, infundibuliformes, 7.8-8.4 cm de largo y $4.5-6.7 \mathrm{~cm}$ de ancho en antesis; pericarpelo globoso a ovoide, alrededor de $2.5 \mathrm{~cm}$ de diámetro, de color púrpura-verdoso, cubierto con podarios poco prominentes, imbricados con primordios foliares triangulares cortos, alrededor de $2 \mathrm{~mm}$ de largo y $2 \mathrm{~mm}$ de ancho en la base, púrpura-verdosos, con tricomas poco densos en las axilas, blanco-amarillentos y espinas suaves y setosas de $1 \mathrm{~cm}$ de largo; tubo receptacular 2.9-3.7 cm de largo, podarios con escamas decurrentes a él, oblongas, de ápice agudo o mucronado, de longitud variable y alrededor de $1 \mathrm{~cm}$ de ancho con tricomas poco densos y espinas; segmentos exteriores del perianto angostamente obovados a espatulados, con el ápice redondeado a agudo o emarginado, alrededor de $3 \mathrm{~cm}$ de largo y de $2 \mathrm{~cm}$ de ancho, verde-purpúreos; segmentos interiores oblongos a espatulados, de hasta $4 \mathrm{~cm}$ de largo y de $2 \mathrm{~cm}$ de ancho, de margen entero, blancos a blancorosados; estambres numerosos, inclusos, insertos en verticilos; filamentos blancoamarillentos; anteras basifijas, amarillentas; estilo alrededor de $5.5 \mathrm{~cm}$ de largo y de $2 \mathrm{~mm}$ de diámetro; lóbulos del estigma 7-11, 7.5-8.5 mm de largo, blancoamarillentos; cámara nectarial semicerrada por la curvatura de los estambres del verticilo inferior, $1.3-1.9 \mathrm{~cm}$ de largo y $0.44-0.64 \mathrm{~mm}$ de ancho, estriada; ovario 0.8$1 \mathrm{~cm}$ de largo y $0.5-0.7 \mathrm{~cm}$ de ancho; fruto globoso a ovoide, dehiscente en la madurez, verde rojizo, anaranjado o de color púrpura, 5-6 cm de diámetro, cubierto por aréolas con numerosas espinas setosas, alrededor de $2 \mathrm{~cm}$ de largo, blancoamarillentas a amarillas, caducas en la madurez, pulpa roja, anaranjada, verde 0 blanca, dulce; semillas 1.8-2 mm de largo y 1.25-1.35 mm de ancho, ovoides, negras, opacas, células de la testa isodiamétricas, con paredes periclinales convexas, sin intersticios, con aspecto finamente verrucoso. 
Fenología. Florece de febrero a marzo, fructifica durante abril, mayo y junio.

Hábitat. Matorral xerófilo en altitudes cercanas a los $1800 \mathrm{~m}$ y en bosque tropical caducifolio en altitudes desde el nivel del mar hasta cerca de los $1600 \mathrm{~m}$.

Distribución. Stenocereus queretaroensis está ampliamente distribuida en Colima, Estado de México, Guanajuato, Jalisco, Michoacán, Nayarit, Querétaro y Zacatecas (Fig. 1). Para el estado de Colima se cita del municipio de Colima (Lemus, 1993), sin embargo no existen ejemplares de respaldo en los herbarios consultados. En Guanajuato, Michoacán y Querétaro Stenocereus queretaroensis convive con Myrtillocactus geometrizans (Mart.) Console y varias especies de Opuntia. En Jalisco y Zacatecas se encuentra cohabitando con Pachycereus pecten-aboriginum y algunas veces con $S$. dumortieri (Scheidw.) Buxb. en el bosque tropical caducifolio.

Ejemplares examinados. Zacatecas: mpio. Juchipila, camino de brecha a $5 \mathrm{~km}$ de Pueblo Viejo hacia el Cerro de Piñones, T. Terrazas 458 (CHAPA). Nayarit: mpio. Ahuacatlán, km 85.5 carretera Tepic - Guadalajara, en el pedregal del Ceboruco, H. Bravo 134 (MEXU); mpio. El Nayar, comunidad dentro del área de influencia del proyecto de Aguamilpa, cerca de la cortina de la presa, $H$. J. Arreola-Nava, $R$. Rodríguez, O. Bravo y R. Ramírez D. 1356 (IBUG). Jalisco: mpio. Atengo, 3 km adelante de Soyatlán por el camino al rancho El Trigo, H. J. Arreola-Nava, $R$. Soltero y J. A. Pérez de la Rosa 132 (MEXU); mpio. Atoyac, predio Isla Chica, en la Laguna de Sayula, $2 \mathrm{~km}$ al oeste de Atoyac, H. J. Arreola-Nava y A. Rodríguez C. 162 (ENCB); mpio. Autlán, $8 \mathrm{~km}$ al sur de Autlán, sobre la carretera a La Huerta, J. Rzedowski y R. McVaugh 1289 (ENCB); mpio. Chapala, cerca de Chapala, H. Bravo $s / n$ (MEXU); mpio. Guadalajara a $1 \mathrm{~km}$ adelante de la desviación hacia Los Camachos por la carretera hacia Saltillo, H. J. Arreola-Nava y C. Nepote 1428 (MEXU); mpio. Juchitlán, $8 \mathrm{~km}$ al noroeste de Juchitlán por la carretera a Tecolotlán, F. J. Santana M. y B. Benz 5646 (IBUG); mpio. La Barca, rivera norte de la Laguna de Chapala, carretera Ocotlán - Jamay, a $9 \mathrm{~km}$ al este de La Barca, H. J. ArreolaNava, P. Hernández y S. González 1095 (IBUG), 1096 (IBUG); mpio. Tepatitlán, $17 \mathrm{~km}$ de Nido de Águilas, $1 \mathrm{~km}$ antes de llegar a la desviación hacia Lagunillas, carretera Tepatitlán - Yahualica, H. J. Arreola-Nava, H. Bravo y L. Scheinvar 63 (MEXU); mpio. Yahualica, cañon del Río Verde $28 \mathrm{~km}$ al sur de Yahualica, J. Rzedowski y R. McVaugh 1275 (ENCB); mpio Zacoalco, carretera Zacoalco Sayula, H. J. Arreola-Nava, E. Sánchez y R. Rodríguez 1342 (IBUG); mpio. Zapopan, carretera Guadalajara - Saltillo, $\mathrm{km} 21$ a $1 \mathrm{~km}$ antes de llegar a la desviación a Ixcatán, H. J. Arreola-Nava y J. Alba 1511 (CHAPA), 1512 (CHAPA). Michoacán: mpio. Benito Juárez, cerca de Pariácuaro, carretera Zitácuaro Huetamo, J. C. Soto Núñez 6388 (IEB); mpio. Churintzio, Zináparo, J. N. Labat s/n (MEXU); 1593 (ENCB); mpio. Jiquilpan, 5 km West of Jiquilpan, A. C. Gibson y $B$. Prigge 3724 (RSA). Guanajuato: mpio. Comonfort, Presa Blanca a $8 \mathrm{~km}$ al sur de Comonfort hacia Celaya, R. T. Bárcenas y M. M. Meade 606 (IEB, MEXU); mpio. Huanímaro, $15 \mathrm{~km}$ al oeste de Guarapo sobre la carretera a Huanímaro, $R$. $T$. 
Bárcenas 636 (MEXU); mpio. León, límite entre Guanajuato y Jalisco sobre la carretera León - Ojuelos, R. T. Bárcenas 451 (MEXU); mpio. León, $5 \mathrm{~km}$ al norte de la carretera Irapuato - León sobre la desviación a Comanjilla, R. T. Bárcenas y M. M. Meade 986 (MEXU); mpio. Manuel Doblado, El Pitayo, 57 km desde La Piedad sobre la carretera a Cuerámaro, R. T. Bárcenas 659 (MEXU); mpio. Tarimoro, La Estancia de San José, $18 \mathrm{~km}$ al sur de Celaya sobre la carretera a Acámbaro, R. T. Bárcenas 612 (IEB, MEXU); mpio. Uriangato, cerca de $10 \mathrm{~km}$ al sur de Yuriria, sobre la carretera a Morelia, R. T. Bárcenas y E. Russman 630 (IEB); $12 \mathrm{~km}$ al oeste de Irapuato, sobre la carretera a La Piedad, H. Bravo y H. SánchezMejorada s/n (MEXU); mpio. Valle de Santiago, cráter lago Hoya del Rincón de Parangueo, $7 \mathrm{~km}$ al oeste de Valle de Santiago, L. I. Aguilera 182 (CHAPA). Querétaro: mpio. Querétaro, camino a Tlacote, desviación hacia Valle Verde, E. Sánchez, Chávez y Lagunas 31 (IEB, MEXU), 32 (IEB, MEXU); mpio. Querétaro, $\mathrm{km} 8$ camino rumbo a Tlacote, desde el entronque con la carretera Querétaro - San Luis Potosí, H. J. Arreola-Nava, A. Orduño y M. A. Martínez-Colín 1529 (CHAPA), 1531 (CHAPA), 1532 (CHAPA); mpio. Querétaro, $13.5 \mathrm{~km}$ al norte de la ciudad de Querétaro, S. Arias y S. Gama 716 (MEXU). Estado de México: mpio. de Santo Tomás de los Plátanos, Malpaís de Ixtapatongo, H. Sánchez-Mejorada 2741 (MEXU).

Stenocereus quevedonis (J. G. Ortega) Bravo, Cact. Suc. Mex. 17: 119. 1972. Lemaireocereus quevedonis J. G. Ortega, Mex. Forest. 6: 1. 1928.

Tipo: México, Sinaloa, Guamúchil (no localizado). Neotipo (aquí designado): México, Michoacán, mpio. La Huacana, desviación La Huacana - Arriaga. 8 km en el camino de Zicuirán - Nuevo Churumuco, 1850'40", 12007'52.3", 13 mayo 1998, H. J. Arreola-Nava, T. Terrazas, S. Arias y R. Herrera 1519 (IBUG).

Árboles candelabriformes de hasta $6 \mathrm{~m}$ de alto; tronco definido, corto, de hasta $50 \mathrm{~cm}$ de largo y $20-25 \mathrm{~cm}$ de diámetro; ramas numerosas, erectas, con pocas ramas secundarias, 2.5-3.5 de largo y $10-15 \mathrm{~cm}$ de diámetro, verdes claras; cavidades de mucílago no evidentes en corte transversal del tallo: costillas 7-9, redondeadas en sección transversal, rectas en sección longitudinal, alrededor de $1.5 \mathrm{~cm}$ de alto y de $2 \mathrm{~cm}$ de ancho en la base, con una constricción horizontal entre las aréolas de la misma costilla; aréolas distantes entre sí 2-2.5 cm, circulares, 7$10 \mathrm{~mm}$ de diámetro, con tricomas glandulares, con el tiempo se cae la glándula y dejan sólo la parte del tricoma no glandular, por lo que no se forma una costra; espinas radiales 9-13, de hasta $1.5 \mathrm{~cm}$ de largo, aciculares a subuladas, blancas, grisáceas con la edad; espinas centrales 3-7, una de ellas más larga ubicada entre las otras, subuladas, con la base ensanchada, fuertes, de hasta $4 \mathrm{~cm}$ de largo, las otras miden hasta $3 \mathrm{~cm}$ de largo, blancas, después grises; flores en la región subapical o lateral, nocturnas, abiertas hasta la mañana siguiente, infundibuliformes, 6.5-8.5 cm de largo y $3.6-5 \mathrm{~cm}$ de ancho en antesis; pericarpelo globoso, alrededor de $1 \mathrm{~cm}$ de diámetro, verde claro, con primordios foliares pequeños, triangulares 
a obovados, cortos, alrededor de $2 \mathrm{~mm}$ de largo y de ancho en la base, verdosos con tricomas blanco-amarillentos en las axilas; tubo receptacular con podarios provistos de escamas oblongas, con el ápice espatulado a mucronado, de longitud variable de hasta $4 \mathrm{~cm}$ y de $6 \mathrm{~mm}$ de ancho, sin espinas; segmentos exteriores del perianto angostamente obovados a espatulados, con el ápice redondeado a agudo, de aproximadamente $2.5 \mathrm{~cm}$ de largo y de $1.5 \mathrm{~cm}$ de ancho, en el haz de color blanco-amarillentos y en el envés con tintes verdosos; segmentos interiores oblongos a espatulados, ápice mucronado a apiculado, de margen entero, alrededor de $3 \mathrm{~cm}$ de largo y de $1.5 \mathrm{~cm}$ de ancho, blancos a amarillentos o rosados; estambres numerosos, inclusos, insertos en la base del perianto en series verticiladas; filamentos blanco-amarillentos; anteras basifijas, amarillentas; estilo alrededor de $4 \mathrm{~cm}$ de largo y de $1.5 \mathrm{~mm}$ de diámetro, blanco-verdoso; lóbulos del estigma 812 , 5.9-8.9 mm de largo, blanco-amarillento; cámara nectarial semiabierta por la base curvada de los estambres del verticilo inferior, $1.1-1.5 \mathrm{~cm}$ de largo y $4.8-6.8 \mathrm{~cm}$ de ancho, estriada; ovario $0.9-1.1 \mathrm{~cm}$ de largo y $7-9 \mathrm{~mm}$ de ancho; fruto globoso a ovoide, verde a verde-rojizo, $5-6 \mathrm{~cm}$ de diámetro, cubierto por aréolas con numerosas espinas setosas, $1.5-2 \mathrm{~cm}$ de largo, blancas, caducas en la madurez, pulpa roja, de sabor dulce; semillas 1.8-2 mm de largo y 1.3-1.5 mm de ancho, obovoides, negras, opacas, células de la testa isodiamétricas, con paredes periclinales convexas, sin intersticios, con aspecto verrucoso.

Fenología. Florece durante abril, fructifica en mayo y junio.

Hábitat. Vive en matorral xerófilo y bosque tropical caducifolio en altitudes de 200 a $500 \mathrm{~m}$.

Distribución. Stenocereus quevedonis fue descrita por González-Ortega de la estación Guamúchil, Sinaloa. Actualmente no se ha encontrado en esta localidad, ya que gran parte de los terrenos están destinados a los distritos de riego. En Michoacán su área de distribución es amplia en la región de la presa del Infiernillo (Fig. 1). Stenocereus quevedonis es muy abundante y conviven con ella $S$. chrysocarpus, S. fricii, S. standleyi, Pachycereus marginatus y P. militaris.

Ejemplares examinados. Michoacán: mpio. Arteaga, $2.3 \mathrm{~km}$ del poblado Palo Pintado sobre la carretara Pátzcuaro - Playa Azul, H. J. Arreola-Nava, T. Terrazas y S. Arias 1573 (CHAPA); mpio. Churumuco de Morelos, Nuevo Churumuco, S. Arias y cols. 684 (MEXU); mpio. La Huacana, carretera cuatro Caminos - Arteaga, $\mathrm{km} \mathrm{174,} \mathrm{orilla} \mathrm{de} \mathrm{la} \mathrm{presa} \mathrm{El} \mathrm{Infiernillo,} \mathrm{H.} \mathrm{J.} \mathrm{Arreola-Nava,} \mathrm{E.} \mathrm{Villegas} \mathrm{y} \mathrm{S.} \mathrm{Arias}$ 1397 (IBUG); mpio. Nueva Italia de Ruiz, desviación La Huacana - Arriaga 6 km en el camino de Zicuirán - Nuevo Churumuco, H. J. Arreola-Nava, T. Terrazas y S. Arias 1514 (CHAPA), 1515 (CHAPA); T. Terrazas, H. J. Arreola-Nava y S. Arias 382 (CHAPA); mpio. Nueva Italia de Ruiz, $2 \mathrm{~km}$ South of San Pedro Barajas, on Highway 57 between Cuatro Caminos and Presa El Infiernillo, A. C. Gibson y B. 
Prigge 3713 (RSA); mpio. Nueva Italia de Ruiz, desviación La Huacana - Arriaga, $8 \mathrm{~km}$ en el camino Zicuirán - Nuevo Churumuco, H. J. Arreola-Nava, T. Terrazas, S. Arias y R. Herrera 1518 (CHAPA), 1519 (CHAPA); mpio. Nueva Italia de Ruiz, desviación La Huacana - Arriaga, 10 km en el camino Zicuirán - Nuevo Churumuco, H. J. Arreola-Nava, T. Terrazas, S. Arias y R. Herrera 1516 (CHAPA), 1517 (CHAPA); mpio. indefinido, cerca de la presa El Infiernillo, H. Sánchez-Mejorada 710502 (MEXU).

Stenocereus thurberi (Engelm.) Buxb., Bot. Stud. 12: 92.1961.

Cereus thurberi Engelm., Am. Journ. Sci. II, 17: 234. 1854.

Pilocereus thurberi (Engelm.) Rümpler, in C. F. Förster. Handb. Cact. ed. 2. 689. 1885.

Lemaireocereus thurberi (Engelm.) Britton et Rose, Cactaceae 2: 97. 1920. Neolemaireocereus thurberi (Engelm.) Backeb., Jahrb. Kakt. 10: 1944.

Marshallocereus thurberi (Engelm.) Backeb., Cact. Succ. J. (USA) 23: 121. 1951.

Tipo: México, Sonora, 1851, G. Thurber s/n (lectotipo MO).

Árboles de hasta $7 \mathrm{~m}$ de alto; tronco no definido o corto, hasta de $30 \mathrm{~cm}$ de largo, 20-30 cm de diámetro; ramas numerosas, que rara vez se vuelven a ramificar, robustas en la base, casi todas de la misma longitud, 0.7-3.5 $\mathrm{m}$ de largo y 10-25 cm de diámetro, verdes claras; cavidades de mucílago evidentes en corte transversal del tallo; costillas 12-21, redondeadas en sección transversal, rectas en sección longitudinal, $1-1.5 \mathrm{~cm}$ de altura y alrededor de $1.5 \mathrm{~cm}$ de ancho en la base, con una constricción horizontal entre las aréolas de la misma costilla; aréolas distantes entre sí $1-3 \mathrm{~cm}$, redondeadas, $1-1.5 \mathrm{~cm}$ de diámetro, con tricomas glandulares que le confieren color marrón a negro; espinas radiales 7-9, aciculares, con la base ligeramente bulbosa, $0.7-1.5 \mathrm{~cm}$ de largo, negras, grisáceas con la edad; espinas centrales 1-3, aciculares, alrededor de $1.5 \mathrm{~cm}$ de largo, la inferior más larga, de hasta $5 \mathrm{~cm}$ de largo, negras, con el tiempo grisáceas; flores subapicales 0 laterales, nocturnas, infundibuliformes, $5.6-8.6 \mathrm{~cm}$ de largo y $3.2-4.8 \mathrm{~cm}$ de ancho en antesis; pericarpelo globoso, alrededor de $1.5 \mathrm{~cm}$ de diámetro, verde claro, cubierto con podarios poco prominentes que tienen primordios foliares triangulares a deltoideos, cortos, de hasta $1.5 \mathrm{~mm}$ de largo y ancho en la base, verdes con tintes rojizos, con tricomas de color marrón oscuro y a veces algunas espinas setosas; tubo receptacular alrededor de $3 \mathrm{~cm}$ de largo, podarios con escamas oblongas, de ápice agudo a espatulado, de tamaño variable y alrededor de $8 \mathrm{~mm}$ de ancho, a veces con algunas espinas setosas blancas de hasta $1 \mathrm{~cm}$ de largo; segmentos exteriores del perianto oblongos a espatulados, con el ápice redondeado a agudo, de margen entero, verdes y en el haz amarillo-verdosos, de hasta de $2.3 \mathrm{~cm}$ de largo y de $1 \mathrm{~cm}$ de ancho; segmentos interiores oblongos a espatulados con el ápice mucronado a apiculado de margen entero, amarillo-verdosos, de hasta $3 \mathrm{~cm}$ de largo y de $1.5 \mathrm{~cm}$ de ancho; estambres numerosos, dispuestos en series verticiladas; filamentos blanco-verdosos; anteras basifijas, amarillas; estilo alrededor 
de $3.5 \mathrm{~cm}$ de largo y de $2 \mathrm{~mm}$ de diámetro; lóbulos del estigma 8-10, 7-9 mm de largo, blanco-amarillentos; cámara nectarial semicerrada por la curvatura de los estambres del verticilo inferior, $1-1.6 \mathrm{~cm}$ de largo y $0.5-0.9 \mathrm{~cm}$ de ancho, estriada; ovario 8-10 mm de largo y de ancho; fruto globoso a ovoide, dehiscente en la madurez por una abertura irregular, verde a rojizo, $3.5-6 \mathrm{~cm}$ de diámetro, cubierto por aréolas, alrededor de $1 \mathrm{~cm}$ de diámetro, con numerosas espinas setosas, alrededor de $2 \mathrm{~cm}$ de largo, blanco-rojizas a negruzcas, caducas en la madurez, pulpa roja, de sabor dulce; semillas 2.2-2.4 mm de largo y 1.5-1.7 mm de ancho, anchamente ovoides, negras, brillantes, células de la testa isodiamétricas, sus paredes ligeramente convexas, casi planas, sin intersticios entre ellas.

Fenología. Florece durante abril y mayo, fructifica en mayo y junio.

Hábitat. El tipo de vegetación donde prospera es el matorral xerófilo, aunque ocasionalmente se puede encontrar en el bosque tropical caducifolio. Habita desde el nivel del mar hasta los $350 \mathrm{~m}$.

Distribución. En el centro y sur de la península de Baja California, parte central de Sinaloa, el estado de Sonora, algunas localidades de Chihuahua, hacia el norte hasta Arizona en los Estados Unidos (Fig. 1). En Baja California crece con otras especies de cactáceas columnares como Pachycereus pecten-aboriginum, $P$. pringlei (S. Watson) Britton et Rose y Stenocereus gummosus (Engelm.) A. C. Gibson et K. E. Horak. En Sonora cohabita con Carnegiea gigantea (Engelm.) Britton et Rose, Pachycereus pecten-aboriginum, P. pringlei, P. schottii (Engelm.) D. R. Hunt, Stenocereus alamosensis y $S$. gummosus. En Sinaloa convive con Pachycereus pecten-aboriginum, P. schottii y S. alamosensis.

Ejemplares examinados. Estados UnIDOs. Arizona: Pima County, Puerto Blanco Mountains, R. H. Peebles y H. W. Parker 14799 (ARIZ); Pima County, 1 mile east of Bates Well, L. Benson 9900 (ARIZ); Organ Pipe Cactus National Monument; 2.4 miles $W$ of Arizona Highway 85 on Puerto Blanco drive, ca. 2 miles NW of Luckville, R. Felger y D. B. Fenn 89-224 (ARIZ); Roskruge Mountains, 2.8 miles northwest of Pescadero Mountain, (photograph), A. C. Gibson y K. E. Horak 1991 (ARIZ). MÉXICO. Baja California Sur: mpio. Cabo San Lucas, extreme southwest between Todos Santos and Cabo San Lucas, in the area between Colonial Plutarco Elias Calles and Las Barrancas, R. Spjut 5393 (MEXU); mpio. Cabo San Lucas, $15 \mathrm{~km}$ al este de Cabo San Lucas, cerro al norte de la playa de Sta. María, J. Ahuatzin Tónix s/n (MEXU); mpio. Ciudad Constitución, Ejido No. 5 al noreste de Ciudad Constitución, R. Encarnación s/n (MEXU); mpio. La Paz, Melitón Albañez, carretera No. 19 La Paz - Los Cabos, 25.7 km sobre la desviación hacia Melitón Albañez, T. Terrazas, S. Arias y A. Orduño 470 (CHAPA); mpio. Loreto, Isla Coronado, cerca de Loreto, L. Scheinvar, N. Kiostenko y E. David 2274 (MEXU); mpio. Loreto, km 24.5 carretera Ciudad Constitución - San Carlos, T. Terrazas, S. Arias y A. Orduño 486 (CHAPA); mpio. Loreto, vicinity of La Victoria, eastern 


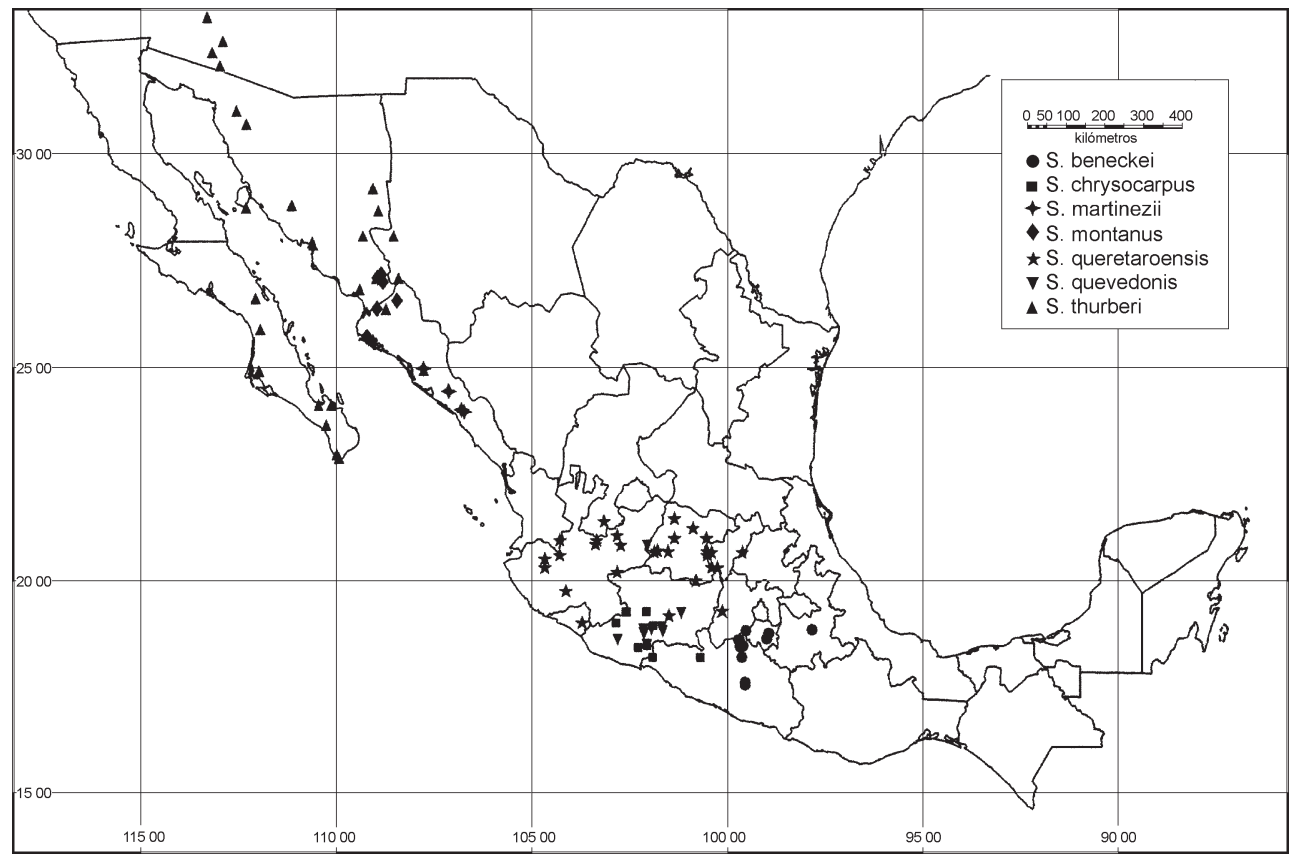

Fig.1. Mapa de distribución de las especies de Stenocereus con aréolas morenas.

escarpment of Sierra de La Giganta southwest of Notri, A. Carter y A. Medellín L. s/n (ENCB); mpio. Loreto, $50 \mathrm{~km}$ al este de Villa Insurgentes, W. López Forment $s / n$ (MEXU). Chihuahua: mpio. Batopilas, $12 \mathrm{~km}$ de Batopilas sobre la orilla de la carretera, F. Simón y M. A. Martínez-Alfaro s/n (MEXU); mpio. Ocampo, $1 \mathrm{~km} \mathrm{~W}$ of boundary of Parque Nacional Cascada de Baseseachic, $R$. Spellenberg, $R$. Corral y E. Estrada C. s/n (MEXU). Sonora: mpio. Álamos, km 9.5 carretera Álamos - San Bernardo, H. J. Arreola-Nava, T. Terrazas y S. Arias 1546 (CHAPA); mpio. Carbó, 0.5 miles south of Los Chinos (50 miles by road north of Hermosillo), R. S. Felger 3274 (ENCB); mpio. Empalme, km 90 carretera Cd. Obregón - Guaymas en la desviación hacia Guásimas, $3 \mathrm{~km}$ sobre la desviación a 200 m de la población de Guásimas, H. J. Arreola-Nava, T. Terrazas y S. Arias 1541 (CHAPA), 1543 (CHAPA), 1545 (CHAPA); mpio. Guaymas, 2 miles North of San Carlos Bay, P. C. Hutchinson 2457 (MEXU); mpio. Guaymas, Bahía de San Carlos, H. Bravo 105 (MEXU); mpio. Santa Ana, Santa Ana, H. Bravo s/n (MEXU). Sinaloa: mpio. El Fuerte, Sindicatura Nochicahui, La Constancia, J. González-Ortega 344 (MEXU); mpio. El Fuerte, $1 \mathrm{~km}$ de Ladesoto, carretera Navojoa - Los Mochis en el entronque hacia El Fuerte 48 km, H. J. Arreola-Nava, T. Terrazas y S. Arias 1554 (CHAPA), 1555 (CHAPA); mpio. Huatabampo, km. 127.5 carretera Navojoa - Los Mochis, 
desviación a Huatabampo $1.5 \mathrm{~km}$, H. J. Arreola-Nava, T. Terrazas y S. Arias 1552 (CHAPA), 1553 (CHAPA); mpio. Los Mochis, Bahía de San Ignacio, al sur del Cerro Cabezón, $35 \mathrm{~km}$ al sureste de Los Mochis, M. Medina C. 2283 (ENCB, IEB, MEXU); mpio. Mocorito, cerca de Mocorito, H. Bravo s/n (MEXU); mpio. Mocorito, Caimanero, cerros $5 \mathrm{~km}$ al oeste de Caimanero, R. Vega y G. Bojórquez 2441 (ENCB).

\section{AGRADECIMIENTOS}

Se agradece a la Comisión Nacional para el Conocimiento y Uso de la Biodiversidad (CONABIO P-L074 otorgado a T. T.) por el apoyo brindado para la realización del trabajo de campo y a la Universidad de Guadalajara que proporcionó una beca (PROMEP-SEP-VDG98-13-01) para realizar estudios de maestría a la primera autora. Agradecemos la colaboración del Biól. Enrique Bermúdez para la elaboración del mapa de distribución, a los herbarios ARIZ, CHAPA, ENCB, IBUG, IEB, MEXU, RSA y SD por el acceso al material consultado y los comentarios críticos del Dr. Roberto Kiesling y de un revisor anónimo.

\section{LITERATURA CITADA}

Arreola-Nava, H. J. 2000. Sistemática de las especies de Stenocereus (A. Berger) Riccob. con aréolas morenas (Cactoideae-Cactaceae). Tesis de Maestría. Colegio de Postgraduados. Montecillo, Estado de México. 103 pp.

Barthlott W. y D. R. Hunt. 1993. Cactaceae In: Kubitzki, K. J. Rohwer y V. Bittrich (eds.). The families and genera of vascular plants. Vol. II. Flowering plants. Dicotyledons. Springer-Verlag. Berlin-Heidelberg. pp. 161-197.

Bravo H., H. 1972. Nuevas cactáceas mexicanas. Cact. Suc. Mex. 17: 115-119.

Bravo H., H. 1978. Las cactáceas de México. Vol. I. 2a ed. Universidad Nacional Autónoma de México. México, D.F. 743 pp.

Bravo H., H. y T. MacDougall. 1956. Una nueva cactácea del occidente de México. An. Inst. Biol. Méx. 27: 316-320.

Britton, N. L. y J. N. Rose. 1920. The Cactaceae. Vol. II, 2a ed. Dover Publications, Inc. Nueva York. 241 pp.

Buxbaum, F. 1961. Die Entwicklungslinien der Tribus Pachycereae F. Buxb. (CactaceaeCereoideae). Bot. Stud. 12:1-105.

Gibson, A. C. 1990. The systematics and evolution of subtribe Stenocereinae. 8. Organ pipe cactus and its closest relatives. Cact. Succ. J. (U.S.A.) 62: 13-24.

Gibson, A. C. y K. E. Horak. 1978. Systematic anatomy and phylogeny of Mexican columnar cacti. Ann. Missouri Bot. Gard. 65: 999-1057.

Gibson, A. C., K. C. Spencer, R. Bajaj y J. L. MacLaughlin. 1986. The ever changing landscape of the cactus systematics. Ann. Missouri Bot. Gard. 73: 532-555.

González-Ortega, J. 1928. Lemaireocereus quevedonis. México Forestal 6: 1.

González-Ortega, J. 1929. Flora indígena de Sinaloa. Imprenta de la Escuela Preparatoria. Mazatlán, Sinaloa. s/p. 
Acta Botanica Mexicana (2003), 64: 1-18

Hunt, D. R. 1999. CITES. Cactaceae checklist. 2a ed. Royal Botanic Gardens Kew and International Organization for Succulent Plant Study. Lemous Limited. Milborne Port. $315 \mathrm{pp}$.

Lemus, S. J. 1993. Las pitayas de Colima. Universidad de Colima. Colima. 35 pp.

Sánchez-Mejorada, H. 1972. Stenocereus chrysocarpus, una nueva especie de Michoacán. Cact. Suc. Mex. 17: 95-98.

Recibido en septiembre de 2000. Aceptado en julio de 2003. 